\title{
Endoscopic examinations of free flap perfusion in the head and neck region using red-excited Indocyanine Green Sven Zhorzel ${ }^{* 1}$, Herbert Stepp ${ }^{2}$, Miriam Havel ${ }^{1}$, Waseem Jerjes ${ }^{3}$, Tahwinder Upile ${ }^{3}$, Colin Hopper ${ }^{3}$, Ulrich Harréus ${ }^{1}$ and Christian S Betz ${ }^{1}$
}

\begin{abstract}
Address: ${ }^{1}$ Department of Otorhinolaryngology, Head \& Neck Surgery, Ludwig Maximilian University Munich, Marchioninistr, Munich, Germany, ${ }^{2}$ Laser-Research Laboratory, LIFE Center, Ludwig Maximilian University, Marchioninistr. 23, 81377 Munich, Germany and ${ }^{3}$ Department of Oral and Maxillofacial Surgery, University College London Hospital, Mortimer Market, London WC1E 6AU, UK

* Corresponding author
\end{abstract}

from Ist Scientific Meeting of the Head and Neck Optical Diagnostics Society

London, UK. 14 March 2009

Published: 28 July 2009

Head \& Neck Oncology 2009, I(Suppl I):P29 doi:I0.II86/I758-3284-I-SI-P29

This abstract is available from: http://www.headandneckoncology.org/content/I/SI/P29

(c) 2009 Zhorzel et al; licensee BioMed Central Ltd.

\section{Objective}

Malfunction of microvascular anastomoses is regarded as the main reason for failure of free tissue transfer. It was the aim of the current investigation to prove the feasibility and to explore the clinical benefit of endoscopically guided free flap perfusion measurements in the head and neck region using red-excited Indocyanine Green (ICG).

\section{Methods}

25 patients who underwent reconstructive surgery including free tissue transfer to the head and neck region took part in this study. Each participant underwent 3 ICG-angiographies (intraoperatively, $24 \mathrm{hrs}$ and $72 \mathrm{hrs}$ postoperatively). The obtained data were evaluated online and offline on PC, and the results compared to the clinical outcome.

\section{Results}

There were no partial or complete losses of transplants. Two flaps with an early arterial failure were successfully salvaged by revision surgery. The ICG-angiographies were tolerated well. The gain of fluorescence was delayed in the transplanted tissue when compared to the surrounding tissue, whereas the final maximum fluorescence intensities were comparable. The two flaps with the initial compromise in perfusion showed relative fluorescence maxima (transplant vs. surrounding) of $33 \%$ or $37 \%$, respectively, whereas these values lay above $64 \%$ for all other examinations.

\section{Conclusion}

It was possible to prove the feasibility of endoscopic ICG angiographies in patients with free tissue transfer to the upper aerodigestive tract. The method is easy to perform and there were no adverse events. Especially in difficult situations (e.g. questionable Doppler signals, flaps situated far down in the pharynx...) the method seems to be a welcome adjunct to conventional screening. 